\title{
Periodic Review Inventory Policy for Non-Instantaneous Deteriorating Items with Time Dependent Deterioration Rate
}

\author{
Anwesha Samanta \\ Department of Statistics University of Calcutta, Kolkata, India \\ Anisam87@gmail.com \\ Manisha Pal \\ Department of Statistics University of Calcutta, Kolkata, India \\ manishapal2@gmail.com
}

\begin{abstract}
The paper studies a periodic review inventory model with no shortages and different demand rates during pre- and post- deterioration periods. Deterioration of units start after a fixed time interval, and the deterioration rate is time dependent. The model determines the optimal reorder interval and the optimal order quantity so as to minimize the total cost per unit length of an inventory cycle. An extension of the model to include price discount has been further considered. Numerical examples are presented to illustrate the model and a sensitivity analysis is also reported.
\end{abstract}

Keywords: Periodic review inventory model; Non-instantaneous deterioration, Time dependent deterioration.

\section{Introduction}

Traditional inventory models assume that depletion from stock is caused only by the arrival of demands. However, in reality, many physical products like volatile liquids, agricultural items, films, blood, drugs, fashion goods, electrical components etc. undergo deterioration through evaporation, spoilage, dryness etc. during their normal storage period. As a result, while developing inventory policies for such products, the loss due to deterioration cannot be ignored. The earliest work along this line is due to Ghare and Schrader (1963), who developed the EOQ model for an exponentially decaying inventory. Thereafter, many authors discussed different inventory models for deteriorating items, like Covert and Philip (1973) extended the model for variable rate of deterioration assuming two parameter Weibull distribution, Philip (1974) generalized EOQ model for items with Weibull distribution.

Generally, it is assumed that the deterioration occurs as soon as the items arrive in inventory. However, in real life, most items retain their quality or original condition for a certain span of time before deteriorating. This phenomenon has been termed as 'noninstantaneous deterioration' by $\mathrm{Wu}$ et al. (2006), and can be commonly observed in products like fruits, vegetables and fashion items. For such items the assumption that the deterioration starts from the instant of arrival in stock may cause retailers to make inappropriate replenishment policies. Lin and Shi (1999) classified inventory models into two categories, viz. decay models and finite lifetime models. Castro and Alfa (2004) proposed a lifetime replacement policy in discrete time for a single unit system. Ouyang et al. (2006) developed an inventory model for non-instantaneous deteriorating items with permissible delay in payments. Chang et al. (2010) developed optimal replenishment 
policies for non-instantaneous deteriorating items with stock-dependent demand. In most of these studies deterioration rate has been assumed to be independent of time.

In this paper we consider an inventory model for non-instantaneous deteriorating items when the deterioration rate is a general function of time. Demand is assumed to be uniform, but the demand rate decreases when the items start to deteriorate. The paper is organized as follows. Section 2 defines the assumptions made and the notations used. Section 3 analyses the model. In Section 4 we introduce price discount in the model. Some numerical examples are given and a study of the sensitivity of the model to change in model parameters is carried out in Section 5. Finally, in Section 6, we make some concluding remarks on our study.

\section{Assumptions and Notations}

We make the following assumptions in our model:

1) There is no lead time.

2) Shortages are not allowed.

3) Deterioration rate is a general function of time.

4) The item considered in inventory is of the Non-instantaneous deteriorating type, i.e. it maintains its freshness for a fixed time interval before deteriorating.

5) Demand occurs at a uniform rate. The demand rate during pre-deterioration period is greater than that in the post-deterioration period.

The following notations have been used in the study:

$D_{1}$ : Pre-deterioration demand rate.

$D_{2}$ : Post-deterioration demand rate, $\mathrm{D}_{1} \geq \mathrm{D}_{2}$.

$\theta(t)$ : Deterioration rate..

$T$ : Length of a reorder interval.

$Q:$ Optimal order quantity.

$\mu$ : Length of pre- deterioration period, $0<\mu \leq T$.

$C_{S}$ :Ordering cost per order, independent of the order quantity.

$C_{1}$ : Purchase cost per unit.

$C_{2}$ : Deterioration cost per unit deteriorating.

$p:$ a fraction such that $p C_{1}$ denotes the carrying cost per item per unit time

$I(t)$ : Inventory level at time $t$.

\section{The Model and its Analysis}

The inventory policy is to place an order for $Q$ units at the beginning of each reorder interval of length $T$. 
Consider the reorder interval $(0, T)$. Since demand rate is $D_{1}$ in the interval $(0, \mu)$ and $D_{2}$ in the interval $(\mu, T)$, and depletion from stock occurs due to both demand and deterioration in the latter interval, the inventory level $I(t)$ satisfies the following differential equations:

$$
\begin{array}{ll}
\frac{d I(t)}{d t}=-D_{1}, & 0 \leq t \leq \mu \\
\frac{d I(t)}{d t}+\theta(t) I(t)=-D_{2} & \mu \leq t \leq T, D_{1} \geq D_{2} .
\end{array}
$$

The boundary conditions are $I(0)=Q$ and $I(T)=0$, which give

$$
\begin{array}{rlrl}
I(t) & =Q-D_{1} t, & & 0 \leq t \leq \mu \\
& =D_{2} \int_{t}^{T} \exp \left(\int_{t}^{y} \theta(x) d x\right) d y, & \mu \leq t \leq T
\end{array}
$$

From (1) and (2), we have

$$
Q=D_{1} \mu+D_{2} \int_{\mu}^{T} \exp \left(\int_{\mu}^{y} \theta(x) d x\right) d y
$$

Equation (3) gives a relationship between the order quantity $Q$ and $T$. Hence, we have only one independent decision variable. Let us take it to be $T$.

\section{Cost Function:}

The different components of the cost function over the interval $(0, T)$ are as follows:

1) Ordering cost $=C_{s}$

2) Purchase cost $=P=C_{1} Q=C_{1} D_{1} \mu+C_{1} D_{2} \int_{\mu}^{T} \exp \left(\int_{\mu}^{y} \theta(x) d x\right) d y$

3) Deterioration cost $=D^{*}=C_{2} \int_{\mu}^{T} \theta(t) I(t) d t$

$$
=C_{2} D_{2} \int_{\mu}^{T} \int_{t}^{T} \theta(t) \exp \left(\int_{t}^{y} \theta(x) d x\right) d y d t
$$

4) Holding cost $=H=C_{1} p \int_{0}^{T} I(t) d t$

$$
\begin{aligned}
=C_{1} p\left[\frac{D_{1} \mu^{2}}{2}+D_{2} \int_{0}^{\mu} \int_{\mu}^{T} \exp \left(\int_{\mu}^{y} \theta(x) d x\right) d y d t+\right. \\
\left.D_{2} \int_{\mu}^{T} \int_{t}^{T} \exp \left(\int_{t}^{y} \theta(x) d x\right) d y d t\right]
\end{aligned}
$$

Since the length of the cycle is a decision variable, we consider the cost per unit length of a cycle. Let it be denoted by $C(T)$.

Then,

$$
\begin{aligned}
C(T)= & \frac{1}{T}\left[C_{s}+P+D^{*}+H\right] \\
=\frac{1}{T}[ & C_{s}+C_{1} D_{1} \mu+C_{1} D_{2} \int_{\mu}^{T} \exp \left(\int_{\mu}^{y} \theta(x) d x\right) d y+ \\
& \quad C_{2} D_{2} \int_{\mu}^{T} \int_{t}^{T} \theta(t) \exp \left(\int_{t}^{y} \theta(x) d x\right) d y d t+C_{1} \mathrm{p} \frac{D_{1} \mu^{2}}{2}
\end{aligned}
$$




$$
\begin{aligned}
& +C_{1} p D_{2} \int_{0}^{\mu} \int_{\mu}^{T} \exp \left(\int_{\mu}^{y} \theta(x) d x\right) d y d t \\
& \left.+C_{1} p D_{2} \int_{\mu}^{T} \int_{t}^{T} \exp \left(\int_{t}^{y} \theta(x) d x\right) d y d t\right] \\
& =\frac{N(T)}{T}, \text { say, }
\end{aligned}
$$

\section{Solution Procedure:}

The optimal value of $T$ that minimises $C(T)$ is a solution of the equation

$$
\frac{\partial C(T)}{\partial T}=0
$$

which gives

$$
\begin{gathered}
C(T)=C_{1} D_{2} \exp \left(\int_{\mu}^{T} \theta(t) d t\right)+C_{2} D_{2} \int_{\mu}^{T} \theta(t) \exp \left(\int_{t}^{T} \theta(x) d x\right) d t+ \\
C_{1} p D_{2} \int_{0}^{\mu} \exp \left(\int_{\mu}^{T} \theta(x) d x\right) d t+C_{1} p D_{2} \int_{\mu}^{T} \exp \left(\int_{t}^{T} \theta(x) d x\right) d t
\end{gathered}
$$

\section{Special Cases:}

Case $1: \theta(t)=\theta$, for all $t \geq \mu$.

In this case, the order quantity $Q$ is related to $T$ according to the following equation:

$$
Q=D_{1} \mu+\frac{D_{2}}{\theta}\left(\mathrm{e}^{\theta(T-\mu)}-1\right)
$$

The expression for $C(T)$ is obtained as:

where

$$
C(T)=\frac{1}{T}\left[C_{0}+H_{1} e^{\theta(T-\mu)}-H_{2}(T-\mu)\right],
$$

$$
\begin{aligned}
& C_{0}=C_{S}+C_{1} D_{1} \mu+C_{1} p \frac{D_{1} \mu^{2}}{2}-\frac{D_{2}}{\theta}\left(C_{1}+C_{2}\right)-C_{1} p \frac{D_{2}}{\theta^{2}}(\mu \theta+1) \\
& H_{1}=\frac{D_{2}}{\theta}\left(C_{1}+C_{2}\right)+C_{1} \mathrm{p} \frac{D_{2}}{\theta^{2}}(\mu \theta+1) \\
& H_{2}=C_{2} D_{2}+C_{1} p \frac{D_{2}}{\theta}
\end{aligned}
$$

and (10) gives

$$
e^{\theta(T-\mu)}(\theta T-1)=\frac{C_{0}+H_{2} \mu}{H_{1}}, \quad T \geq \mu
$$

Theorem 1: The optimal value of $T$ is

$$
\begin{aligned}
T=\mu, \text { if } \theta \mu-1 \geq \frac{C_{0}+H_{2} \mu}{H_{1}} \\
=T^{*}, \text { if } \theta \mu-1<\frac{C_{0}+H_{2} \mu}{H_{1}},
\end{aligned}
$$

where $T^{*}$ is the value of $T$ satisfying (10). 
Proof: We have

$$
\frac{\partial C(T)}{\partial T}=e^{\theta(T-\mu)}(\theta T-1)-\frac{C_{0}+H_{2} \mu}{H_{1}}
$$

Let, $f(T)=e^{\theta(T-\mu)}(\theta T-1)$.

$f(T)$ is an increasing function of $T$, with $f(\mu)=\theta \mu$-1. Hence, when $\theta \mu-1 \geq \frac{C_{0}+H_{2} \mu}{H_{1}}$, that is $f(\mu) \geq \frac{C_{0}+H_{2} \mu}{H_{1}}$, we have $\frac{\partial C(T)}{\partial T} \geq 0$ for $T \geq \mu$. Thus, in the range $T \geq \mu$, optimum value of $T$ is $\mu$.

When $\theta \mu-1<\frac{C_{0}+H_{2} \mu}{H_{1}}, f(\mu)<\frac{C_{0}+H_{2} \mu}{H_{1}}$ and $f(\infty)>\frac{C_{0}+H_{2} \mu}{H_{1}}$. Hence, the curve $y=f(T)$ cuts the line $y=\frac{C_{0}+H_{2} \mu}{H_{1}}$ at a point $T=T^{*} \in(0, \infty)$. Therefore,

$$
\frac{\partial C(T)}{\partial T} \leq 0 \text { for } T \leq T^{*} \text { and } \frac{\partial C(T)}{\partial T} \geq 0 \text { for } T \geq T^{*} \text {. }
$$

Case 2 : $\theta(t)=\theta(t-\mu)$ for all $t \geq \mu$..

In this case, the order quantity $Q$ is related to $T$ according to the following equation:

$$
Q=D_{1} \mu+D_{2} \int_{0}^{T-\mu} \exp \left(\frac{\theta}{2} z^{2}\right) d z
$$

The expression for $C(T)$ is given by

where

$$
C(T)=\frac{1}{\mathrm{~T}}\left[C s+P+D^{*}+H\right],
$$

$$
\begin{aligned}
& P=C_{1} D_{1} \mu+C_{1} D_{2} \int_{0}^{T-\mu} \exp \left(\frac{\theta}{2} z^{2}\right) d z \\
& \begin{aligned}
D^{*}=C_{2} D_{2} \int_{0}^{T-\mu} \exp \left(\frac{\theta}{2} z^{2}\right) d z-C_{2} D_{2}(T-\mu) \\
H=\mathrm{C}_{1} \mathrm{p}\left[\frac{\mathrm{D} 1 \mu 2}{2}+\mathrm{D}_{2}\left(\mu-\sqrt{\frac{\pi}{2 \theta}}\right) \int_{0}^{T-\mu} \exp \left(\frac{\theta}{2} z^{2}\right) d z\right. \\
+D_{2} \sqrt{\frac{2 \pi}{\theta}} \int_{0}^{T-\mu} \exp \left(\frac{\theta}{2} z^{2}\right) \phi(\mathrm{z} \sqrt{ } \theta) d z .
\end{aligned}
\end{aligned}
$$

Thus, we can write

$$
C(T)=\frac{1}{T}\left[A_{1}-A_{2}(T-\mu)+A_{3} \int_{0}^{T-\mu} \exp \left(\frac{\theta}{2} z^{2}\right) d z+A_{4} \int_{0}^{T-\mu} \exp \left(\frac{\theta}{2} z^{2}\right) \phi(\mathrm{z} \sqrt{ } \theta) d z\right],
$$

where $A_{1}=C_{1} D_{1} \mu+C_{1} p \frac{D_{1} \mu^{2}}{2}+C s, A_{2}=C_{2} D_{2}, A_{3}=\left(C_{1}+C_{2}\right) D_{2}+C_{1} p D_{2}\left(\mu-\sqrt{\frac{\pi}{2 \theta}}\right)$,

$$
A_{4}=C_{1} p D_{2} \sqrt{ } \frac{2 \pi}{\theta} \text {. }
$$

The optimal value of $T$ that minimises $C(T)$ must satisfy

$$
\frac{\partial C(T)}{\partial T}=0,
$$

which gives 


$$
C(T)=-A_{2}+A_{3} \exp \left(\frac{\theta}{2}(T-\mu)^{2}\right)+A_{4} \exp \left(\frac{\theta}{2}(T-\mu)^{2}\right) \phi((T-\mu) \sqrt{\theta} .
$$

Now,

$$
\begin{gathered}
\frac{\partial^{2}}{\partial T^{2}} C(T)=\exp \left(\frac{\theta}{2}(T-\mu)^{2}\right)\left[\left\{A_{3} \theta(T-\mu)+A_{4} \phi\{(T-\mu) \sqrt{\theta}\} \theta(T-\mu)+A_{4} \sqrt{\theta} \phi\{(T-\mu) \sqrt{\theta}\}\right]\right. \\
\geq 0, \text { for } T \geq \mu .
\end{gathered}
$$

Hence the cost function $C(T)$ is convex of $T$. This means that any solution of (18) will give the optimal value of $T$.

\section{Model with Price Discount}

It is a common experience that when the inventory manager orders a large quantity of goods he is given a discount on his purchase. Different inventory models with price discount have been studied by many authors. See, for example, Ardalan (1994), Wee and Yu (1997), Pal and Dutta (2007), Panda et al. (2009), Cardanas-Barron et al. (2010).

Let,

$$
\begin{aligned}
C_{1} & =C_{1 U}, \text { if } Q<b \\
& =C_{1 D}, \text { if } Q \geq b,
\end{aligned}
$$

where $C_{1 U}>C_{1 D}$.

We assume that $b \geq D_{1} \mu$. This is because, from (3), $Q<b$ is equivalent to

$$
\int_{\mu}^{T} \exp \left(\int_{\mu}^{y} \theta(x) d x\right) d y<\frac{b-D_{1} \mu}{D_{2}} .
$$

LHS of (19) $\geq 0$ for $T \geq \mu$, but RHS $<0$ unless $b \geq D_{1} \mu$.

Let the cost function be denoted by

$$
\begin{aligned}
C(Q) & =C_{U}(Q), \quad \text { if } Q<b \\
& =C_{D}(Q), \text { if } Q \geq b,
\end{aligned}
$$

Since $C(Q)$ is a linear function of $C_{1}$, and $C_{1 U}>C_{1 D}$, it follows that $C_{U}(Q)>C_{D}(Q)$ for all $Q>0$.

From (3) it is obvious that $Q$ is a one-to-one increasing function of $T$. Hence, we can write $T=g(Q)$, for some increasing function $g($.$) . We, therefore, have that if T_{0}=g(b)$, then $Q<b$ is equivalent to $T<T_{0}$.

Thus,

(i) for $\theta(t)=\theta$, independent of $t, T_{0}=\mu+\frac{1}{\theta} \ln \left[1+\frac{\theta}{D_{2}}\left(b-D_{1} \mu\right)\right]$;

(ii) for $\theta(t)=\theta(t-\mu), T_{0}$ is obtained by solving the equation

$$
\int_{0}^{T_{0}-\mu} \exp \left(\frac{\theta}{2} z^{2}\right) d z=\frac{b-D_{1} \mu}{D_{2}} .
$$


We can, therefore, write

$$
\begin{aligned}
C(T) & =C_{U}(T), \text { for } \mu \leq T<T_{0} \\
& =C_{D}(T), \text { for } T \geq T_{0} .
\end{aligned}
$$

Further, $C_{U}(T)>C_{D}(T)$ for all $T \geq \mu$.

To find the optimal value of $T$, and hence of $Q$, that minimizes $C(T)$, we compare the minimum values of $C_{U}(T)$ and $C_{D}(T)$. If $C_{U}(T)$ and $C_{D}(T)$ be strictly convex in $T$, for $\mu \leq$ $T<\infty$, with minimum at $T_{U}$ and $T_{D}$ respectively, then we can use the following algorithm to find the optimal value of $T$ that minimizes $C(T)$.

\section{Algorithm 1:}

Step 1: Find $T_{D}$ minimising $C_{D}(T)$.

(i) If $T_{D} \geq T_{0}, T_{D}$ is the optimal value of $T$ that minimizes $C(T)$.

(ii) If $T_{D}<T_{0}$, go to Step 2 .

Step 2: Find $T_{U}$ minimising $C_{U}(T)$, and compute $C_{U}\left(T_{U}\right)$ and $C_{D}\left(T_{0}\right)$.

(i) If $\left.C_{D}\left(T_{0}\right)\right) \geq C_{U}\left(T_{U}\right), T=T_{U}$ minimizes $C(T)$.

(ii) If $\left.C_{D}\left(T_{0}\right)\right) \leq C_{U}\left(T_{U}\right), T=T_{0}$ minimizes $C(T)$.

The algorithm follows from the following arguments:

(a) When $T_{D} \geq T_{0}$,

$$
\begin{aligned}
& C(T)=C_{D}(T) \geq C_{D}\left(T_{D}\right)=C\left(T_{D}\right), \text { for } T \geq T_{0} \\
& C(T)=C_{U}(T) \geq C_{D}(T) \geq C_{D}\left(T_{D}\right)=C\left(T_{D}\right), \text { for } \mu \leq T<T_{0} .
\end{aligned}
$$

Thus, $C(T) \geq C\left(T_{D}\right)$, for all $T \geq \mu$.

(b) When $T_{D}<T_{0}, C_{D}(T)$ is an increasing function of $T$ for $T \geq T_{0}$. Hence,

$$
\min _{T \geq T_{0}} C_{D}(T)=C_{D}\left(T_{0}\right) \text {. }
$$

Then,

(i) for $\left.C_{D}\left(T_{0}\right)\right) \geq C_{U}\left(T_{U}\right)$,

$$
\begin{aligned}
& C(T)=C_{D}(T) \geq C_{D}\left(T_{0}\right) \geq C_{U}\left(T_{U}\right)=C\left(T_{U}\right), \text { for } T \geq T_{0} \\
& C(T)=C_{U}(T) \geq C_{U}\left(T_{U}\right)=C\left(T_{U}\right), \text { for } \mu \leq T<T_{0} .
\end{aligned}
$$

Hence, $C(T) \geq C\left(T_{U}\right)$, for all $T \geq \mu$;

(ii) for $\left.C_{D}\left(T_{0}\right)\right) \leq C_{U}\left(T_{U}\right)$,

$$
\begin{aligned}
& C(T)=C_{D}(T) \geq C_{D}\left(T_{0}\right)=C\left(T_{0}\right), \text { for } T \geq T_{0} \\
& C(T)=C_{U}(T) \geq C_{D}\left(T_{0}\right)=C\left(T_{0}\right), \text { for } \mu \leq T<T_{0} .
\end{aligned}
$$

Hence, $C(T) \geq C\left(T_{0}\right)$, for all $T \geq \mu$. 


\section{Numerical Examples and Sensitivity Analysis}

Example 1: Consider an item that can maintain its freshness for 6 months and then starts deteriorating with a deteriorating rate 0.2 per unit time. The demand rate for the item is 300units per unit time before deterioration but decreases to 200units per unit time when the item starts to deteriorate. The costs are: $C_{S}=$ Rs. $100, C_{1}=$ Rs. 12 per unit, $p=1 / 3, C_{2}=$ Rs. 5 per unit deteriorating. We have to determine the optimal order quantity and the optimal reorder interval.

Here, $\theta \mu-1=0.2$ and $\frac{C_{0}+H_{2} \mu}{H_{1}}=0.908>\theta \mu-1$.

Hence optimal $T$ is obtained by solving the equation (16), which gives $T=8.0275$ months. The optimal order quantity $Q$ is, therefore, 2050.026 units and the minimum cost per unit length of an inventory cycle is Rs. 6649.996.

Example 2: Let us consider an item that can maintain its freshness for 2 months and then starts to deteriorate with a deteriorating rate $0.02(t-\mu)$ per unit time. The demand for the item is 80 units per unit time in its fresh state, but reduces to 50 units per unit time when deterioration starts. The ordering cost is Rs. 100 per unit, purchase cost is Re. 1 per unit, deterioration cost is Rs. 0.75 per unit and the carrying cost is a proportion 0.6 of the cost per unit.

Solving equation (18) we get optimal $T=3.500713$ months, optimal order quantity = 235.5977 units and minimum cost per unit length of a cycle is Rs. 159.0462.

Example 3: Suppose in example 2 the purchase cost is Rs. 3 per unit if $Q<200$ units, and is Rs. 2 per unit for $Q \geq 200$ units.

Here $T_{0}=2.8$ months. Since the cost function is convex in $T$, algorithm 1 can be applied.

We get $T_{D}=3.069$ months, which is greater than $T_{0}$. Hence the inventory manager should accept the discount offer. The optimum order quantity is then $Q=213.66$ units, and the total cost per month is Rs. 282.9942.

We next examine the sensitivity of the model to a change in the model parameters. For the study, we consider example 2.

Tables 1-5 show the change in the optimal values of $T$ and $Q$ and the percent change in the minimal cost when the value of a parameter changes. 
Periodic Review Inventory Policy for Non-Instantaneous Deteriorating Items with Time Dependent ......

Table 1: Change in the optimal values of $T$ and $Q$, and the percent change in $C(T)$ with change in $C_{1}$

\begin{tabular}{|c|c|c|c|c|}
\hline $\boldsymbol{C}_{\mathbf{1}}$ & $\boldsymbol{T}$ & $\boldsymbol{Q}$ & $\boldsymbol{C}(\boldsymbol{T})$ & $\begin{array}{c}\text { \% change } \\
\text { in } \boldsymbol{C}(\boldsymbol{T})\end{array}$ \\
\hline $\mathbf{1}$ & $\mathbf{3 . 5 0 0 4 7 7}$ & $\mathbf{2 3 5 . 5 9 7 7}$ & $\mathbf{1 5 9 . 0 4 6 2}$ & $\mathbf{0}$ \\
\hline 1.5 & 3.232944 & 221.9581 & 224.1392 & 40.93 \\
\hline 2 & 3.06907 & 213.6559 & 287.5992 & 80.83 \\
\hline 2.5 & 2.970891 & 208.6961 & 351.1999 & 120.82 \\
\hline 3 & 2.902255 & 205.2434 & 414.6293 & 160.70 \\
\hline
\end{tabular}

Table 2: Change in the optimal values of $T$ and $Q$, and the percent change in $C(T)$ with change in $p$

\begin{tabular}{|c|c|c|c|c|}
\hline $\boldsymbol{p}$ & $\boldsymbol{T}$ & $\boldsymbol{Q}$ & $\boldsymbol{C}(\boldsymbol{T})$ & $\begin{array}{c}\text { \% change } \\
\text { in } \boldsymbol{C}(\boldsymbol{T})\end{array}$ \\
\hline 0.5 & 3.730301 & 247.3803 & 148.3024 & -6.76 \\
\hline $\mathbf{0 . 6}$ & $\mathbf{3 . 5 0 0 4 7 7}$ & $\mathbf{2 3 5 . 5 9 7 7}$ & $\mathbf{1 5 9 . 0 4 6 2}$ & 0 \\
\hline 0.7 & 3.31651 & 226.2044 & 169.3587 & 6.48 \\
\hline 0.75 & 3.178974 & 219.2204 & 171.8892 & 8.08 \\
\hline 0.8 & 3.165855 & 218.5555 & 179.3300 & 12.75 \\
\hline
\end{tabular}

Table 3: Change in the optimal values of $T$ and $Q$, and the percent change in $C(T)$ with change in $C_{2}$

\begin{tabular}{|c|c|c|c|c|}
\hline $\boldsymbol{C}_{\mathbf{2}}$ & $\boldsymbol{T}$ & $\boldsymbol{Q}$ & $\boldsymbol{C}(\boldsymbol{T})$ & $\begin{array}{c}\text { \% change } \\
\text { in } \boldsymbol{C}(\boldsymbol{T})\end{array}$ \\
\hline 0.5 & 3.51975 & 236.5718 & 159.314 & 0.17 \\
\hline $\mathbf{0 . 7 5}$ & $\mathbf{3 . 5 0 0 4 7 7}$ & $\mathbf{2 3 5 . 5 9 7 7}$ & $\mathbf{1 5 9 . 0 4 6 2}$ & 0 \\
\hline 1 & 3.493685 & 235.2389 & 159.0845 & 0.02 \\
\hline 2 & 3.467634 & 233.9076 & 159.2308 & 0.12 \\
\hline 3 & 3.443883 & 232.7320 & 159.3928 & 0.21 \\
\hline
\end{tabular}


Table 4: Change in the optimal values of $T$ and $Q$, and the percent change in $C(T)$ with change in $\theta$

\begin{tabular}{|c|c|c|c|c|}
\hline $\boldsymbol{\Theta}$ & $\boldsymbol{T}$ & $\boldsymbol{Q}$ & $\boldsymbol{C}(\boldsymbol{T})$ & $\begin{array}{c}\text { \% change } \\
\text { in } \boldsymbol{C}(\boldsymbol{T})\end{array}$ \\
\hline 0.01 & 3.553233 & 237.9813 & 158.7628 & 0.18 \\
\hline $\mathbf{0 . 0 2}$ & $\mathbf{3 . 5 0 0 4 7 7}$ & $\mathbf{2 3 5 . 5 9 7 7}$ & $\mathbf{1 5 9 . 0 4 6 2}$ & 0 \\
\hline 0.03 & 3.46152 & 233.8640 & 159.3166 & 0.17 \\
\hline 0.04 & 3.413477 & 231.6313 & 159.5637 & 0.32 \\
\hline 0.05 & 3.376839 & 229.9407 & 159.7927 & 0.47 \\
\hline
\end{tabular}

Table 5: Change in the optimal values of $T$ and $Q$, and the percent change in $C(T)$ with change in $\mu$

\begin{tabular}{|c|c|c|c|c|}
\hline $\boldsymbol{\mu}$ & $\boldsymbol{T}$ & $\boldsymbol{Q}$ & $\boldsymbol{C}(\boldsymbol{T})$ & $\begin{array}{c}\text { \% change } \\
\text { in } \boldsymbol{C}(\boldsymbol{T})\end{array}$ \\
\hline 1 & 2.896916 & 255.9844 & 142.5872 & -10.35 \\
\hline $\mathbf{2}$ & $\mathbf{3 . 5 0 0 4 7 7}$ & $\mathbf{2 3 5 . 5 9 7 7}$ & $\mathbf{1 5 9 . 0 4 6 2}$ & 0 \\
\hline 3 & 4.16786 & 218.6569 & 177.7867 & 11.78 \\
\hline 4 & 4.713061 & 195.7130 & 198.7911 & 24.99 \\
\hline 5 & 5.598705 & 189.9720 & 218.8527 & 37.60 \\
\hline
\end{tabular}

The above tables indicate that the model is highly sensitive to change in $C_{1}$, the purchase cost per unit, moderately sensitive to changes in $p$ and $\mu$, but is quite insensitive to changes in the deterioration $\operatorname{cost} C_{2}$ and $\theta$.

\section{Conclusion}

The paper studies a periodic review inventory model for a non-instantaneous deteriorating item that has a general deteriorating time distribution. Withdrawal from stock occurs at a uniform rate, but the rate decreases when items in stock start to deteriorate. The situation where discount is offered to the inventory manager on a purchase of a large quantity of the item is also discussed.

The model has scope for extension by considering demand to be dependent on time and stock, as well as on the deterioration rate. 


\section{References}

1. Ardalan, A. (1994). Optimal prices and order quantities when temporary price discounts result on increase in demand, European Journal of Operational Research, 72(1), 52-61.

2. Cardenas-Barron, L. E, Smith, N. R. and Goyal, S. K. (2010). Optimal Order size to take advantage of a one-time discount offer with allowed backorders, Applied Mathematical Modeling, 34, 1642-1652.

3. Castro, I.T. and Alfa, A.S. (2004). Lifetime replacement policy in discrete time for a single unit system, Reliability Engineering and Safety, 84, 103-111.

4. Chang, Chun-Tao, Teng, Jinn-Tsair and Goyal, S.K. (2010). Optimal replenishment policies for non-instantaneous deteriorating items with stockdependent demand, International Journal of Production Economics, 123, 62-68.

5. Covert, R.P. and Philip, G.C. (1973). An EOQ model for items with Weibull distribution deterioration, Amer. Inst. Ind. Eng. Trans., 5, 323-326.

6. Ghare, P.M. and Schrader, G.F. (1963): A model for exponentially decaying inventories, International Journal of Production Research, 21, 449-460.

7. Lui, L. and Shi, D. (1999). An (s,S) model for inventory with exponential lifetimes and renewal demands, Naval Research Logistics, 46, 39-56.

8. Ouyang, L.Y., Wu, K.S. and Yang, C.T. (2006). A study on an inventory model for non-instantaneous deteriorating items with permissible delay in payments, Computer and Industrial Engineering, 51, 637-651.

9. Pal, M. and Dutta, S.S. (2007). An inventory model with single price break and convexity of cost function, Opsearch, 44(2).

10. Panda, S., Saha, S. and Basu, M. (2009). An EOQ model for perishable products with discounted selling price and stock dependent demand, Central European Journal of Operational Research, 17(1), 31-53.

11. Philip, G.C. (1974). A generalized EOQ model for items with Weibull distribution deterioration, AIIE Transactions, 6(2), 159-162.

12. Wee, H. M. and Yu, J. (1997). A deteriorating inventory model with a temporary price discount, International Journal of Production Economics, 53, 81-90.

13. Wu, K.S., Ouyang, L.Y. and Yang, C.T. (2006). An optimal replenishment policy for non-instantaneous deteriorating items with stock-dependent demand and partial backlogging, International Journal of Production Economics, 101, 369384. 